\title{
Comparison of Nasoendoscopic Findings and Quality of Life between Type II Diabetics and Non-Diabetics with Chronic Rhinosinusitis
}

\author{
Oluyomi Samuel Ayodele ${ }^{1 *}$, Kola John Olarinoye ${ }^{2}$, Sulyman Biodun Alabi ${ }^{1}$, Emmanuel \\ Foluwasayo Ologe ${ }^{1}$ \\ ${ }^{\text {I}}$ Ear, Nose and Throat Department, University of Ilorin Teaching Hospital, Ilorin, Nigeria \\ ${ }^{2}$ Endocrinology unit, Department of Medicine, University of Ilorin Teaching Hospital, Ilorin, Nigeria \\ *Corresponding Author: Dr. Oluyomi Samuel Ayodele, Ear, Nose and Throat Department, University \\ of Ilorin Teaching Hospital, PMB 1459, Ilorin, Nigeria. Email: oluayo4me@yahoo.com
}

\begin{abstract}
Aim: The study is aimed at comparing nasoendoscopic findings and quality of life (QoL) between type II diabetics and non-diabetic adults with chronic rhinosinusitis (CRS).

Methods: Cross-sectional study carried out on 71 cases and 71 controls. QoL measured with sinonasal outcome test (SNOT-22) was obtained. Nasoendoscopy was performed and scored with the Discharge, Inflammation and Polyps/Oedema (DIP) endoscopic scoring system.

Results: Of the 71 type II diabetics with CRS, 40.8\% were males and 59.2\% females. The modal age group was 51 - 60 years. The mean SNOT-22 and DIP scores were 41.6 and 14.3 among type II diabetics with CRS respectively. There was a strong positive correlation between DIP and SNOT-22 scores, however no statistically significant difference in the DIP score and SNOT-22 test between type II diabetics with CRS and non-diabetics with CRS.

Conclusion: DIP score and SNOT-22 test were similar between type II diabetics with CRS and non-diabetics with CRS. There was higher number of type II diabetics and non-diabetics with moderate impact of CRS on QoL using SNOT-22. Type II diabetes is therefore not a predictor of sinonasal outcome in CRS patients. DIP score and SNOT-22 can be routinely used in assessment and management of CRS.
\end{abstract}

Keywords: Chronic rhinosinusitis, Type II Diabetics, Sinonasal outcome test, Nasoendoscopy, DIP score.

\section{INTRODUCTION}

Chronic rhinosinusitis (CRS) is a group of disorders characterised by inflammation of the mucosal lining of the nose and paranasal sinuses for at least 12 consecutive weeks. [1], [2] According to the Clinical Practice Guideline formulated by the Rhinosinusitis Task Force of the American Academy of Otolaryngology, Head and Neck Surgery (AAO-HNS), diagnostic criteria for CRS depends on clinical symptoms and signs which are categorised into minor and major criteria. [2], [3]

The criteria rely on the identification of symptoms to make a diagnosis. The symptoms are divided into major symptoms [nasal discharge (anterior/posterior), nasal congestion, facial pressure or pain, and hyposmia/anosmia] and minor symptoms (headache, fever, halitosis, fatigue, dental pain, cough, ear pain, pressure, and/or fullness).[3] When a patient presents with two major symptoms or one major and two minor symptoms, then it is diagnostic of CRS. [4]

CRS is a common disease worldwide, constituting $78 \%$ of the rhinology cases attending the otolaryngology clinic in West Africa. [5] In Nigeria, rhinosinusitis was reported as the commonest rhinological disorder and second commonest otorhinolaryngological disorder with a prevalence of $7.3 \%$ in a study in North-Western region. [6] Its burden on the quality of life (QoL) of the affected individuals is significant and the disease poses management challenges to the physicians. However, the negative effects of CRS on QoL of the patients have frequently been underestimated. 
Nevertheless, many patients report higher impacts of CRS on QoL dimensions like bodily pain or social functioning compared with other diseases like angina pectoris, pulmonary emphysema or chronic bronchitis. [7] Sinonasal Outcome Test questionnaire (SNOT-22) has been found to be valid and easy to use to in clinical practice to highlight the impact of CRS on the patient's quality of life.[8]

Diabetes Mellitus (DM) is a chronic metabolic disorder characterised by chronic hyperglycaemia, caused by an absolute or relative deficiency in insulin secretion resulting into disorder of carbohydrates, protein and fat metabolism.[9] Type II DM is characterised by insulin resistance in peripheral tissues and an insulin secretory defect of the beta cell. There are at least 5 million Nigerians affected.[9] It is also the most prevalent form, it constitutes about 96\% of people living with diabetes.[10] The prevalence in Nigeria varies from $0.65 \%$ in rural Mangu (North), 6.8\% in Port Harcourt and to $11 \%$ in urban Lagos (South). [9] The world health statistics indicate that Nigeria has the highest number of people living with diabetes in Sub-Sahara Africa with associated high morbidity and mortality. [9]

It is generally believed that DM increases patients' susceptibility to various types of infections (which includes chronic rhinosinusitis) due to the metabolic derangements. [11] However, the relationship between type II diabetes and chronic rhinosinusitis has not been well studied worldwide indicating a gap in knowledge which has led to controversies as to the effect of type II DM (as a systemic disease) on a known Diabetic with chronic rhinosinusitis. [11] [12] The study by Zi Zhang et al [11] appears to be the first to assess the impact of type II DM on CRS in a large clinical cohort. Interestingly, he found that CRS patients with DM were also significantly more likely to have nasal polyps as compared to patients without DM. [11] However, Chung et al [12] found no significant differences between subjects with and those without CRS who were observed in the prevalence of uncomplicated or complicated diabetes.[12] Zhang et al [11] proposed that further studies are needed to objectively assess whether diabetes affects the inflammatory status of the mucosal lining of the nasal cavity and sinuses to facilitate the development of nasal polyps. Similarly, objective measures such as nasal endoscopy scores are needed to further evaluate the impact of DM on CRS as well as to determine whether DM control improves the prognosis of CRS. [11] This study sought to address the gap in knowledge by determining the comparison of the nasoendoscopic findings and health related quality of life assessment between people who have type II diabetes with chronic rhinosinusitis and non-diabetics with chronic rhinosinusitis attending Ear, Nose and Throat (ENT) and diabetes clinics at University of Ilorin Teaching Hospital.

\section{MATERIALS AND MeTHOdS}

This was a hospital based comparative crosssectional analytical study carried out at University of Ilorin Teaching Hospital, Ilorin, Nigeria over a period of 8 months. With a convenience sampling technique, consenting adults with CRS and known type II DM (from both ENT and Diabetes clinics) were recruited after a detailed explanation of the purpose of the study and procedures involved. Similarly, age and sex matched control group was selected from CRS patients who were free of diabetes from ENT clinic. Written informed consents were obtained. With a study proforma, information on the sociodemographic status, history of CRS and type II DM were obtained. The results of fasting blood sugar as at the time of the diagnosis were obtained, documented and formed the basis for recruitment into the diabetic group (FBS $\geq 7.0 \mathrm{mmol} / \mathrm{L}$ ). Clinical diagnosis of CRS was made using the guideline by the Rhinosinusitis Task Force of the American Academy of Otolaryngology, Head and Neck Surgery (AAO-HNS). [13] Clinical assessment of all subjects included ENT examination and nasal endoscopy. The SNOT22 questionnaire was administered on each consenting subject. The 22-item Sinonasal Outcome Test is a validated health status questionnaire, specific to analyse quality of life in sinonasal diseases. [8] It includes assessments of nasal, paranasal and psychological symptoms, and those associated with sleep. The endoscopic findings were scored with the sheet provided for the Discharge, Inflammation and Polyps/oedema (DIP) scoring system. [14] Each category was scored on an 11-point Likert scale from 0 to 10 based on the viewer's overall assessment of the sinonasal mucosa; with a total of 30 points for each nasal cavity. 
Ethical approval was sought and obtained from the Ethical Review Committee (ERC) of the University of Ilorin Teaching Hospital (ERC PAN/2018/11/1844). Statistical analysis was carried out using Statistical Product and Service Solutions (SPSS) version 20. Results were presented in simple tables and figure. Descriptive statistics (mean, standard deviations, frequencies and correlation coefficients) were calculated for all measures. The association between continuous variables and specific outcome variables were tested using student t-test where the assumptions were met; otherwise a non-parametric test was applied such as chi square test. For all statistical studies, $p$ value $<0.05$ was considered as being statistically significant.

\section{RESUltS}

A total of 71 type II diabetics with CRS (cases) and 71 non-diabetics patients with CRS (controls) participated in the study. As shown on Table 1, the age range of cases was between 37 and 88 years with a mean age of 56.69 years \pm 9.42 while the control group had age range from 29 to 84 years with mean age of 54.32 years \pm 13.38. The highest number of cases $27(38.0 \%)$ and controls $24(33.8 \%)$ were found in age group 51-60. The cases comprised of 42 (59.2 $\%)$ females and $29(40.8 \%)$ males with a male to female ratio of 1:1.4. The control group comprised of $41(57.7 \%)$ females and 30 $(42.3 \%)$ males; with a male to female ratio of 1:1.4.

Table1. Age and sex distribution and duration of chronic rhinosinusitis of subjects

\begin{tabular}{|c|c|c|c|c|}
\hline Variables & Case $(\%), n=71$ & Control (\%), n=71 & $\chi^{2} / \mathbf{t}$ & $\rho$ value \\
\hline \multicolumn{5}{|l|}{ Age Groups } \\
\hline$\leq 30$ & $0(0.0)$ & $3(4.2)$ & & \\
\hline $31-40$ & $3(4.2)$ & $8(11.3)$ & & \\
\hline $41-50$ & $11(15.5)$ & $13(18.3)$ & & \\
\hline $51-60$ & $27(38.0)$ & $24(33.8)$ & & \\
\hline $61-70$ & $20(28.2)$ & $18(25.4)$ & & \\
\hline$\geq 71$ & $10(14.1)$ & $5(7.0)$ & $4.001^{\mathrm{y}}$ & 0.549 \\
\hline Mean \pm SD & $56.69 \pm 9.42$ & $54.32 \pm 13.38$ & 1.942 & 0.224 \\
\hline Range & $37-88$ & $29-84$ & & \\
\hline \multicolumn{5}{|l|}{ Gender } \\
\hline Male & $29(40.8)$ & $30(42.3)$ & & \\
\hline Female & $42(59.2)$ & $41(57.7)$ & 0.029 & 0.865 \\
\hline \multicolumn{5}{|c|}{ CRS Duration (Months) } \\
\hline$\leq 48$ & $49(69.0)$ & $57(80.3)$ & & \\
\hline$>48$ & $22(31.0)$ & 14 (19.7) & & \\
\hline Mean \pm SD & $44.28 \pm 40.14$ & $37.98 \pm 37.07$ & 2.382 & 0.123 \\
\hline Range & $4-240$ & $3-240$ & 1.100 & 0.273 \\
\hline
\end{tabular}

$\chi^{2}$ : Chi-square. $t:$ T test. $p$ : test of statistical significance. ${ }^{y}:$ Yates corrected value

Among the cases, CRS ranged from 4 months to 20 years with mean duration of 3 years 8 months and 1 week and 3 days (Mean $=44.28 \pm 40.14$ ). Among the controls, CRS ranged from 3 months to 20 years with mean duration of 3 years, 1 month, 3 weeks, 6 days (Mean $=37.98 \pm 37.07$ ). As presented on Table 1, the duration of CRS was classified into $\leq 48$ months and $>48$ months for both cases and control groups. Among the cases, 49 (69 \%) had symptoms of CRS for 4 months to 4 years while $22(31 \%)$ had symptoms above 4 years. Among the control group, 57 (80.3\%) had symptoms of CRS for 3 months to 4 years while $14(19.7 \%)$ had symptoms above 4 years. There was no statistically significant difference in the duration of CRS between cases and controls ( $p$ value $=$ $0.123)$.
Figure 1 represented the average score of each of the 22 questions on SNOT-22 for all cases (blue) and controls (red). A similar pattern was observed for both case and control groups. As presented on table 2, the mean total quality of life score on sinonasal outcome test was $41.59 \pm$ 19.06 among the cases. And among the controls, a mean total quality of life score of $43.39 \pm$ 20.19 was found. CRS was found to be a moderate problem in $34(47.9 \%)$ cases followed by moderate to severe problem in $28(39.4 \%)$. Severe problem to as bad as it can be and mild problem were found among $8(11.3 \%)$ and 1 (1.4\%) respectively. Similar pattern was found among the control group and there was no statistically significant difference between the cases and controls ( $p$ value $=0.995)$. Results of DIP score showed a mean total score of $14.34 \pm$ 
Comparison of Nasoendoscopic Findings and Quality of Life between Type II Diabetics and NonDiabetics with Chronic Rhinosinusitis

6.41 and $15.20 \pm 6.80$ for cases and controls respectively. Among the cases, $32(45.1 \%)$ had moderate DIP score while $23(32.4 \%)$ and 16 $(22.5 \%)$ had mild and severe DIP score respectively. Similar pattern was found among the control (Table 2). The difference of the DIP scores between the case and control groups was not statistically significant $(p$ value $=0.792)$.

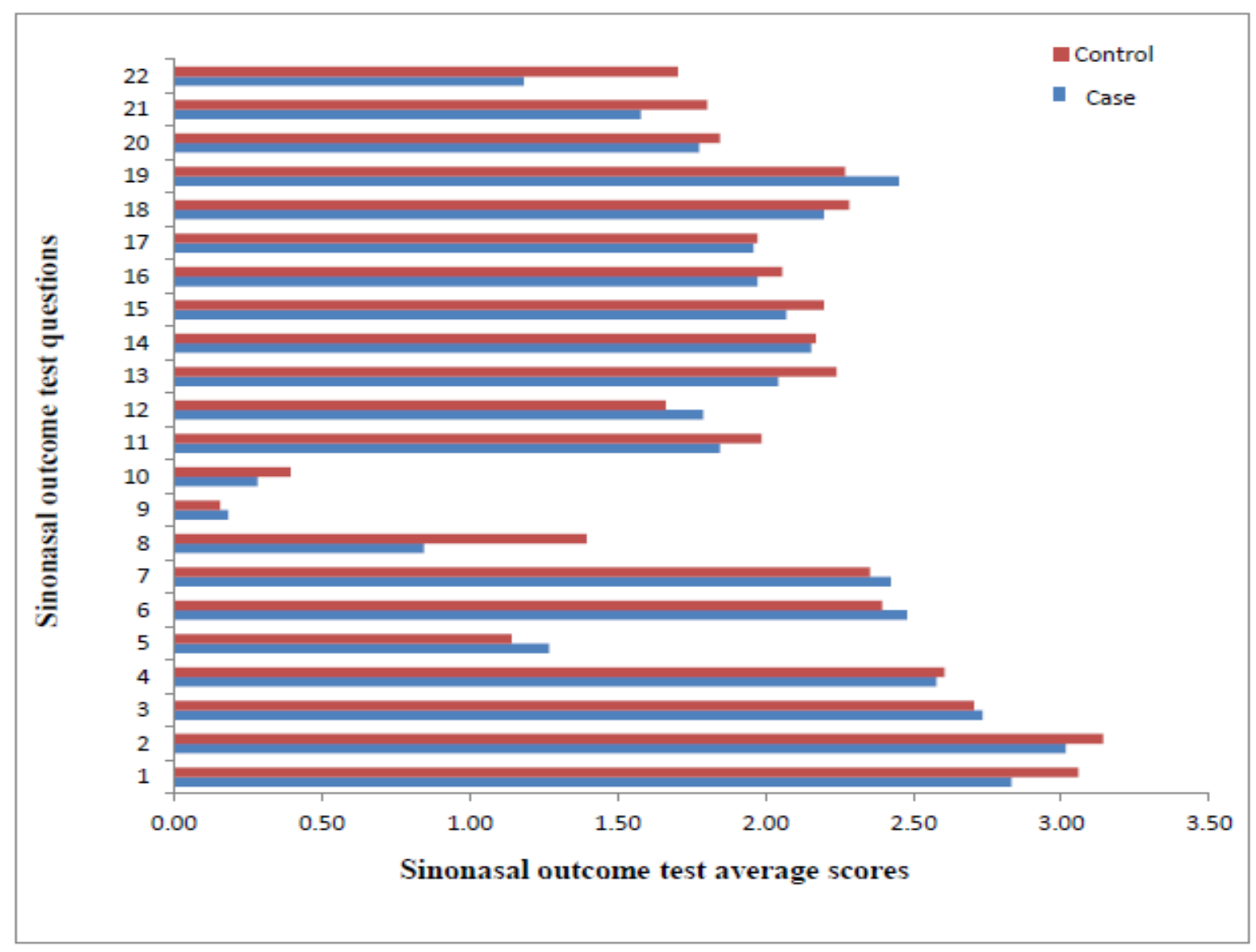

Figure1. Bar chart representing the comparison of SNOT-22 average scores between cases and controls

Table2. Analysis of Sinonasal Outcome Test (SNOT 22) and the of Average Discharge, Inflammatory and Polyps/oedema (DIP) Score in both cases and controls

\begin{tabular}{|c|c|c|c|c|}
\hline Variables & Case $(\%), n=71$ & Control $(\%), n=71$ & $\chi^{2} / \mathrm{t}$ & $\rho$ value \\
\hline \multicolumn{5}{|l|}{ SNOT 22} \\
\hline Mild problem & $1(1.4)$ & $0(0.0)$ & & \\
\hline Moderate problem & $34(47.9)$ & $34(47.9)$ & & \\
\hline Moderate to severe problem & $28(39.4)$ & $27(38.0)$ & & \\
\hline Severe problem to as bad as it can be & $8(11.3)$ & $10(14.1)$ & $0.070^{y}$ & 0.995 \\
\hline Mean \pm SD & $41.59 \pm 19.06$ & $43.39 \pm 20.19$ & -0.547 & 0.585 \\
\hline Range & $10-97$ & 11-86 & & \\
\hline \multicolumn{5}{|l|}{ DIP Score } \\
\hline Mild & $23(32.4)$ & $20(28.1)$ & & \\
\hline Moderate & $32(45.1)$ & $32(45.1)$ & 0.446 & 0.792 \\
\hline Severe & $16(22.5)$ & $19(26.8)$ & -0.774 & 0.440 \\
\hline Mean \pm SD & $14.34 \pm 6.41$ & $15.20 \pm 6.80$ & & \\
\hline Range & $3-26$ & $5-30$ & & \\
\hline
\end{tabular}

$\chi^{2}$ : Chi-square $t:$ Test $p$ : test of statistical significance ${ }^{y}$ : Yates corrected value

All cases who had SNOT-22 score of severe problem to as bad as it can be also had severe score on the DIP nasal endoscopic scoring system (Table 3). A total of $19(67.9 \%)$ of 28

ARC Journal of Diabetes and Endocrinology cases who had a SNOT-22 score of moderate to severe problem also had moderate DIP score. The results among controls were similar. The association between the severity of SNOT-22 
and DIP scores was statistically significant among cases and controls ( $p$ values < 0.05). SNOT-22 score also had a strong positive correlation with average DIP score and was statistically significant: $r_{\mathrm{s}}=0.759, p$ value $=$ 0.000 on Spearman's correlation coefficient $\left(\mathrm{r}_{\mathrm{s}}\right)$.

Table3. Association between Sinonasal Outcome Test and Discharge, Inflammation and Polyps/oedema score in cases and controls

\begin{tabular}{|c|c|c|c|c|c|c|c|c|}
\hline \multirow[t]{2}{*}{ Variables } & \multicolumn{3}{|c|}{ Case $(\%), n=71$} & \multirow[t]{2}{*}{$\chi^{2} / \rho$} & \multicolumn{3}{|c|}{ Control $(\%) n=71$} & \multirow[t]{2}{*}{$\chi^{2} / p$} \\
\hline & Mild & Moderate & Severe & & Mild & Moderate & Severe & \\
\hline \multicolumn{9}{|l|}{ SNOT 22} \\
\hline Mild problem & $1(100.0)$ & $0(0.0)$ & $0(0.0)$ & & $0(0.0)$ & $0(0.0)$ & $0(0.0)$ & \\
\hline Moderate problem & $20(58.9)$ & $13(38.2)$ & $1(2.9)$ & & $19(55.9)$ & $14(41.2)$ & $1(2.9)$ & \\
\hline Moderate to severe problem & $2(7.1)$ & $19(67.9)$ & $7(25.0)$ & & $1(3.7)$ & $16(59.3)$ & $10(37.0)$ & \\
\hline $\begin{array}{l}\text { Severe problem to as bad as } \\
\text { it can be }\end{array}$ & $0(0.0)$ & $0(0.0)$ & $8(100.0)$ & $\begin{array}{l}42.019^{\mathrm{y} /} \\
<\mathbf{0 . 0 0 1}^{*}\end{array}$ & $0(0.0)$ & $2(20.0)$ & $8(80.0)$ & $\begin{array}{l}32.727^{\mathrm{y}} / \\
<0.001 *\end{array}$ \\
\hline
\end{tabular}

$\chi^{2}$ : Chi-square; *: Statistically significant (i.e. p value <0.05); ${ }^{y}:$ Yates corrected value

\section{DISCUSSION}

The highest number of cases found within the age range of 51- 60 was consistent with the study by Adogu et al [15] in Owerri, where the age group most affected was $51-60$ years. This study found a slightly higher female preponderance among the cases $(\mathrm{M}: \mathrm{F}=1: 1.4)$. Sogebi et al, [16] da Lilly-Tariah, [17] and Onotai et al [18] found similar gender distribution. This could be because women are more concerned about their health and thus seek medical attention more promptly and often than men.[19] Adebisi et al, [20] in his study on the glycated haemoglobin and glycaemic control of diabetics in Ilorin, found that fifty-six percent of the study populations were females. Without any comorbidity put into consideration, Fasunla et al [5] and Afolabi et al [21] found a slight male preponderance in their studies on patients presenting with rhinosinusitis.

The duration of CRS as revealed by this study was within the range reported by previous studies where mean duration of CRS ranged between 2 to 6 years. [16], [17], [22], [23] The possible explanations for this high mean duration include the fact that most patients tend to take the symptoms casual as they see it as a common but mere environmental problem. [16] They therefore depend on self-medications until when the disease becomes persistent or manifest with other ominous sequel necessitating presentation in the hospital. [16] This study was carried out in a tertiary institution; most patients would have visited other peripheral health centres before presenting to the specialist clinics.

The results of quality of life assessment using the SNOT-22 questionnaire showed that the mean total quality of life score in the study group was similar to findings among the controls. This finding therefore means that type II diabetes does not worsen the health-related quality of life among patients with chronic rhinosinusitis when compared to non-diabetics with CRS. This is similar to what Zhang et al [11] found in his work on the effect of diabetes mellitus on chronic rhinosinusitis and sinus surgery outcome: the mean scores of SNOT-22 reported for patients with type II diabetes and non-diabetics were $41 \pm 18$ and $40 \pm 23$ respectively. This study found a higher number of participants with moderate impact of CRS on health-related quality of life among the cases and controls with no statistically significant difference between both groups ( $p$ value $=$ 0.902). In a similar study by Boyko and Lipsky, [24] the overall prevalence of self-reported chronic rhinosinusitis was identical for the type II diabetics and non-diabetics. Though, Marambaia et al [25] found that the ones with worse QoL were more frequently affected by DM; however, there was no significant difference between the cases and control group. Zhang et al [11] in USA also found no significant difference between patients with and without DM in the preoperative SNOT-22 scores ( $p$ value=0.926). However, when compared to non-DM patients, CRS patients with type II diabetes had less improvement in QoL from baseline to 6-month postoperative clinic visits. The reason for the high number of subjects with moderate quality of life is likely to be because a large number of patients were already on one form of medical management or the other which might have ameliorated the effect of the clinical features on their QoL as at the time of this study since the QoL measure with SNOT-22 was only based on how patient felt within the last two weeks. [26] With the use of the SNOT-22 questionnaires, previous studies 
on the quality of life of patients with CRS proved that CRS has negatively effects and major impact on the activities of daily living of these patients in comparison to people without the disease or people with other chronic diseases like congestive heart failure and chronic obstructive pulmonary disease. [25] Adegbiji et $a l$, [27] in the study on clinicoepidemiological pattern of rhinosinusitis also found that rhinosinusitis resulted in affectation of quality of life in $81.0 \%$ of the studied population.

All cases that had SNOT-22 score of severe problem to as bad as it can be also had severe score on the DIP nasal endoscopic scoring system in this study. Similarly, a larger percentage $(67.9 \%)$ of cases with moderate to severe problem in SNOT-22 also had moderate DIP score. Spearman's correlation coefficient of average DIP score and other continuous variables revealed that SNOT-22 had strong positive correlation with average DIP score. This means that a high DIP score can depict worsening health-related QoL. Thus, DIP scores and SNOT-22 could be reliable tools for management of chronic rhinosinusitis in resource poor outstations. This agrees with Rudmik et al [28] where endoscopy scores were found to increase as SNOT-22 scores increase. In another study on evaluating the diagnosis of chronic rhinosinusitis based on clinical guidelines and endoscopy, Bhattacharyya et al [29] agreed with the fact that patients who meet symptom criteria with a subjective symptoms score, also need the addition of objective nasal endoscopic examination which will improve diagnostic accuracy of CRS. Thus, nasal endoscopy should remain a mainstay of the objective evaluation of CRS patient. [29], [30]

In the study of a novel sinonasal endoscopy scoring system; DIP score, Durr et al [14] associated SNOT-20 (which has being modified into SNOT-22 by National Comparative Audit of Surgery for Nasal Polyposis and Rhinosinusitis of the Royal College of Surgeons, England [26], [31]) with the endoscopic findings among patients with CRS. They found a contrary result showing no positive correlation between nasal endoscopy score and symptom score, which is also consistent with results from prior studies where SNOT-20 and other previous subjective symptom scores were used.[30], [32] The added questions which made up SNOT-22 are very significant because problems with olfaction and nasal obstruction are directly related to the quality of life of patients with CRS, therapeutic interventions are designed to positively influence these two annoying symptoms. [8] They are therefore likely to correlate well with nasal endoscopic features of discharge, inflammatory and/or polyps/edema. Ryan et al [30] found that symptoms had poor correlation with endoscopic scores but he agreed with this submission that nasal congestion/ obstruction, and hyposmia may be individually predictive of abnormal nasal endoscopy.

\section{CONCLUSiON}

To the best of our literature research, studies done in West African sub region are yet to look into the nasoendoscopic findings and quality of life of type II diabetics with CRS in comparison with non-diabetics with CRS. This study revealed that the results of DIP score and SNOT-22 test were similar between type II diabetics with CRS and non-diabetics with CRS. Similarly, a higher number of type II diabetics and non-diabetics with moderate impact of CRS on their health-related quality of life using SNOT-22 was found. A higher DIP score depicts worse SNOT-22 health related QoL; although, no statistically significant difference in the results of DIP nasoendoscopic score and SNOT-22 between type II diabetics with CRS and non-diabetics with CRS. Therefore, Type II diabetes is therefore not a determinant or predictor of sinonasal outcome in CRS patients. However, DIP nasoendoscopic scoring system and SNOT-22 should be routinely used in the clinical assessment and assessment of healthrelated QoL respectively in the management of CRS patients in an ENT clinic setting.

\section{LIMITATION OF THE STUDY}

A large number of the subjects in this study were already on one form of medical treatment or the other, which might have ameliorated the effect of the clinical features on their QoL as at the time of this study. Similarly, the HRQoL measure with SNOT-22 was only based on how patient felt within the last two weeks, which may not be enough for holistic assessment of the QoL of CRS patients. This study didn't also put into consideration the different types of chronic rhinosinusitis vis-a-vis their effects on QoL of type II diabetics with CRS.

\section{DisCLOSURES}

This research did not receive any specific grant from funding agencies in the public, commercial, or not-for-profit sectors. No conflicting interest as well. 


\section{REFERENCES}

[1] W. J. Fokkens et al., "EPOS 2012: European position paper on rhinosinusitis and nasal polyps. A summary for otorhinolaryngologists.," Rhinology, vol. 50, no. 1, pp. 1-12, 2012.

[2] M. S. Benninger et al., "Adult chronic rhinosinusitis: definitions, diagnosis, epidemiology, and pathophysiology.," Otolaryngol. neck Surg., vol. 129, no. 3 Suppl, pp. S1-32, 2003.

[3] D. C. Lanza and D. W. Kennedy, "Adult rhinosinusitis defined.," Otolaryngol. neck Surg., vol. 117, no. 3 Pt 2, pp. S1-7, 1997.

[4] M. S. Benninger and J. K. Stokken, "Acute Rhinosinusitis: Pathogenesis, Treatment, and Complications," in Cummings Otolaryngology Head and Neck Surgery, 6th ed., P. W. Flint, Ed. Canada: Elsevier Inc., 2015, p. 724.

[5] A. J. Fasunla and O. G. B. Nwaorgu, "Adult Chronic Rhinosinusitis: Spectrum of Clinical Features in a Tertiary Health Institution and Literature Review.," East Cent Afr J Surg, vol. 16, no. 1, pp. 12-18, 2011.

[6] K. R. Iseh and M. Makusidi, "Rhinosinusitis: A retrospective analysis of clinical pattern and outcome in North Western Nigeria.," Ann. Afr. Med., vol. 9, no. 1, pp. 20-26, 2010.

[7] D. G. Becker, "Sinusitis," J. long-term Eff. Med. Implant., vol. 13, no. 3, pp. 175-194, 2003.

[8] C. Hopkins, S. Gillett, R. Slack, V. J. Lund, and J. P. Browne, "Psychometric validity of the 22item Sinonasal Outcome Test.," Clin. Otolaryngol., vol. 34, no. 5, pp. 447-54, 2009.

[9] S. Chineye, Clinical Practice Guidelines for Diabetes Management in Nigeria., 2nd ed. Nigeria: Bekdik, 2013.

[10] O. A. Fasanmade and S. Dagogo-Jack, "Diabetes Care in Nigeria.," Ann. Glob. Heal., vol. 81, no. 6, pp. 821-829, 2015.

[11] Z. Zhang et al., "The effect of diabetes mellitus on chronic rhinosinusitis and sinus surgery outcome.," Int. Forum Allergy Rhinol., vol. 4, no. 4, pp. 315-320, 2014.

[12] S.-D. Chung, P.-Y. Chen, H.-C. Lin, and S.-H. Hung, "Comorbidity profile of chronic rhinosinusitis: A population-based study," Laryngoscope, vol. 124, no. 7, pp. 1536-41., 2014.

[13] R. M. Rosenfeld et al., "Clinical practice guideline (update): Adult sinusitis.," Otolaryngol. neck Surg., vol. 152, no. 2 Suppl, pp. S1-S39, 2015.

[14] M. L. Durr, S. D. Pletcher, A. N. Goldberg, and A. H. Murr, "A novel sinonasal endoscopy scoring system: the discharge, inflammation, and polyps/edema (DIP) score.," Int Forum Allergy Rhinol., vol. 3, no. 1, pp. 66-72., 2013.

[15] P. O. U. Adogu, H. N. Chineke, M. U. Ewuzie, O. O. Enwere, and N. B. Egenti, "The Prevalence and Presentation Pattern of Diabetes Mellitus in Patients at Imo State University Teaching Hospital (IMSUTH) Orlu and Imo State Specialist Hospital (IMSSH) Umuguma Owerri(A 10-Year Retrospective Study).," J. Diabetes Mellit., vol. 5, pp. 49-57., 2015.

[16] O. A. Sogebi and E. A. Oyewole, "Rhinosinusitis: Clinical Features Seen In Sagamu, Nigeria," Internet $J$. Otorhinolaryngol., vol. 6, no. 2, pp. 1-4, 2006.

[17] O. B. da Lilly-Tariah, "Pattern of clinical features of chronic simple rhinosinusitis in Port Harcourt.," Niger. J. Clin. Pract., vol. 9, no. 2, pp. 142-6., 2006.

[18] L. Onotai and U. Oparaodu, "Rhinosinusitis in adult patients: analysis of clinical pattern and outcome of management," Gaz. Med., vol. 5, no. 1, pp. 1-15, 2016.

[19] P. Mc Dounough and V. Walters, "Gender and health: reassessing patterns and explanations," Soc. Sci. Med., vol. 52, pp. 547-559, 2001.

[20] S. A. Adebisi, E. K. Oghagbon, T. M. Akande, and J. K. Olarinoye, "Glycated haemoglobin and glycaemic controls of diabetes in Ilorin," Niger. J. Clin. Pract., vol. 12, no. 1, pp. 87-91, 2009.

[21] O. A. Afolabi et al., "Management and Outcome of Rhinosinusitis in Nigeria," OTO Open, vol. 1, no. 1, pp. 1-7, 2017.

[22] E. Kolo, "The role of Plain Radiographs in the Diagnosis of Chronic Maxillary Rhinosinusitis in Adults.," Afr. Health Sci., vol. 4, pp. 459463, 2012.

[23] S. V Nath, S. James, and N. Suresh, "A prospective study of clinical profile of chronic rhinosinusitis in a tertiary care centre," J. Evol. Med. Dent. Sci, vol. 6, no. 16, pp. 1268-1275, 2017.

[24] E. J. Boyko and B. A. Lipsky, "Infection and Diabetes," in Diabetes in America, 2nd ed., M. I. Harris and C. C. Cowie, Eds. Bethsda: National Institutes of Diabetes, 1995, pp. 485500.

[25] P. P. Marambaia, M. G. Lima, K. P. Santos, G. A. de Machado, M. M. de Sousa, and M. M. E. de Macedo, "Evaluation of the quality of life of patients with chronic rhinosinusitis by means of the SNOT-22 questionnaire.," Brazilian $J$. Otorhinolaryngol., vol. 79, no. 1, pp. 54-8., 2013.

[26] J. F. Piccirillo, M. G. Merritt, and M. L. Richards, "Psychometric and clinimetric validity of the 20-Item Sino-Nasal Outcome Test (SNOT-20).," Otolaryngol. neck surgery., vol. 126, no. 1, pp. 41-7., 2002. 
[27] A. W. Adegbiji, S. K. Aremu, G. O. Akanbi, F. Olatoke, and K. O. Ajite, "Clinicoepidemiological Pattern of Rhinosinusitis in a University Hospital in Ekiti, Nigeria," Glob. J. Otolaryngol., vol. 12, no. 4, pp. 1-6, 2018.

[28] L. Rudmik, Z. M. Soler, J. C. Mace, A. S. Deconde, R. J. Schlosser, and T. L. Smith, "Using preoperative SNOT-22 score to inform patient decision for endoscopic sinus surgery," Laryngoscope, vol. 125, no. 7, pp. 1517-1522, 2015.

[29] N. Bhattacharyya and L. N. Lee, "Evaluating the diagnosis of chronic rhinosinusitis based on clinical guidelines and endoscopy," Otolaryngol. - Head Neck Surg., vol. 143, no. 1, pp. 147-151, 2010.
[30] W. R. Ryan, T. Ramachandra, and P. H. Hwang, "Correlations Between Symptoms, Nasal Endoscopy, and In-Office Computed Tomography in Post-Surgical Chronic Rhinosinusitis Patients," Laryngoscope, vol. 121, pp. 674-678, 2011.

[31] J. F. Piccirillo, D. Edwards, A. Haiduk, C. Yonan, and S. E. Thawley, "Psychometric and Clinimetric Validity of the 31-Item Rhinosinusitis Outcome Measure (RSOM-31)," Am. J. Rhinol., vol. 9, no. 6, pp. 297-306., 1995.

[32] D. S. Birch, H. A. Saleh, T. Wodehouse, I. N. Simpson, and I. S. Mackay, "Assessing the quality of life for patients with chronic rhinosinusitis using the 'Rhinosinusitis Disability Index'.," Rhinology, vol. 39, pp. 191-196., 2001.

Citation: Samuel Oluyomi Ayodele et al. Comparison of Nasoendoscopic Findings and Quality of Life between Type II Diabetics and Non-Diabetics with Chronic Rhinosinusitis. ARC Journal of Diabetes and Endocrinology. 2020; 6(1):14-21. DOI: https://doi.org/10.20431/2455-5983.0601003.

Copyright: (C) 2020 Authors. This is an open-access article distributed under the terms of the Creative Commons Attribution License, which permits unrestricted use, distribution, and reproduction in any medium, provided the original author and source are credited. 